accurately capture cell shape. We postulated that this might be important as $\mathrm{T}$ cells adopt different shapes when scanning for antigen and after recognizing MHC class II-restricted peptides.

Methods We implemented a deep convolutional neural network (DCNN) that accurately identified both cell position and shape. The DCNN output was then analyzed with a tuned convolutional neural network (TNN) to identify distance and cell shape features that best discriminated between different $\mathrm{T}$ cell populations relative to dendritic cells (DCs). We refer to this analysis pipeline as $\mathrm{CDM}^{3}$.

Results In mice, $\mathrm{CDM}^{3}$ discriminated between cognate and non-cognate $\mathrm{T}$ cell interactions with $\mathrm{DCs}$ with a sensitivity and specificity similar to most TPEM measures. In human lupus nephritis, $\mathrm{CDM}^{3}$ both confirmed that myeloid DCs present antigen to $\mathrm{CD}^{+}{ }^{+} \mathrm{T}$ cells in situ and identified plasmacytoid DCs as an important antigen presenting cell in severe inflammation.

Conclusions $\mathrm{CDM}^{3}$ provides a novel tool for quantifying in situ adaptive immune cell networks broadly applicable to the study of human diseases including autoimmunity and cancer. Acknowledgements Funded by the NIH including the Autoimmunity Centers of Excellence.

\section{TD-04 PLAQUENIL DISCONTINUATION IS ASSOCIATED WITH INCREASED DAMAGE ACCUMULATION IN SLE}

${ }^{1}$ Caroline Siegel, ${ }^{1}$ Jennifer Grossman, ${ }^{1}$ Sarah Chen, 'Lori Sahakian, ${ }^{2}$ Michael Gorin, ${ }^{1}$ Maureen McMahon*. 'Divisions of Rheumatology, UCLA David Geffen School of Medicine, Los Angeles, CA, USA; ${ }^{2}$ Ophthalmology, UCLA David Geffen School of Medicine, Los Angeles, CA, USA

\subsection{6/lupus-2018-Ism.123}

Background There is ample evidence demonstrating that hydroxychloroquine (HCQ) prevents lupus flares, increases long-term survival, and protects against irreversible organ damage in SLE (Pons-Estel Arth Care Res 2010). However, in recent years, ophthalmology guidelines have encouraged providers to limit cumulative HCQ exposure to prevent ocular toxicity. We examined whether a) HCQ use in our UCLA cohort has changed over time and b) whether patients who discontinue HCQ accumulate damage at a different rate than continuous or never users.

Methods SLE subjects participating in our longitudinal 'Biomarkers of Atherosclerosis' carotid ultrasound cohort were evaluated. Medication use (and reasons for disuse) were recorded at cohort entry, and at each subsequent study visit.

303 SLE subjects participated in the baseline study (20042006), 229 in the 2nd follow-up (2008-2010), and 102 in the 3rd follow-up exam (2015-present).

For continuity, SLICC Damage Index (SDI) was measured at baseline, 3 years, and 5 years after cohort entry.

Results At baseline, 69.8\% of patients were taking HCQ, $62.1 \%$ at first follow-up, and $58.6 \%$ at second follow-up $(\mathrm{p}=\mathrm{ns})$. Patient preference and physician preference were the most common reasons for HCQ non-use. Less than 3\% of subjects who discontinued HCQ did so due to concern for eye toxicity at baseline and first follow-up, but this increased to $7.4 \%$ at second follow-up.
We compared SDI at baseline in patients who were never HCQ users $(n=29)$, vs previous users $(n=93)$, vs continuously users $(n=181)$. We found evidence of SLE-related damage $(\mathrm{SDI} \geq 1)$ in $75.9 \%$ of never HCQ users, $74.1 \%$ of previous users, and $52.4 \%$ of subjects on HCQ at baseline $(\mathrm{p}=0.001$, Chi-Square). At 5 years, we found $\mathrm{SDI} \geq 1$ in $94.7 \%$ of never HCQ users, $82.7 \%$ of previous users vs $68.2 \%$ of subjects on HCQ at baseline $(\mathrm{p}=0.004$, Chi-Square).

Conclusions Although the frequency HCQ use in our longitudinal SLE cohort has decreased slightly over the last 13 years, the percentage of subjects who discontinued HCQ due to eyerelated eye toxicity has increased recently. Further studies will be required to determine whether this increase is due solely to cumulative HCQ dose vs more frequent detection because of adherence to ophthalmology testing guidelines vs earlier detection due to more sensitive testing modalities. Our data also demonstrate that damage accumulation is similar in subjects who previously used HCQ and those who never used, and higher compared to continuous users, suggesting continuous HCQ use may be important for damage prevention in SLE.

\section{TD-05 DYNAMIC CONTRAST ENHANCED MRI (DCE-MRI) DEMONSTRATES HIPPOCAMPUS PERMEABILITY IN SLE}

${ }^{1}$ Meggan Mackay*, ${ }^{2}$ Joan M Chi, ${ }^{2}$ Angela Hoang, ${ }^{2}$ Katherine Cheng, ${ }^{3}$ Jana Ivanidze, ${ }^{1}$ Bruce Volpe, ${ }^{1}$ Cynthia Aranow, ${ }^{1}$ Betty Diamond, ${ }^{4}$ Pina C Sanelli. ${ }^{1}$ Feinstein Institute for Medical Research, Center for Auroimmune, Musculoskeletal and Hematopoietic Diseases, Manhasset, NY; ' Department of Radiology, Donald and Barbara Zucker School of Medicine at Hofstra/Northwell, Manhasset, NY; ${ }^{3}$ Department of Radiology, Weill Cornell Medical College, New York, NY; ${ }^{4}$ Feinstein Institute for Medical Research, The Center for Health Innovations and Outcomes Research, Manhasset, NY

\subsection{6/lupus-2018-Ism.124}

Background Cross-reactive, anti-dsDNA/N-methyl D-aspartate receptor antibodies (DNRAb) have been associated with cognitive impairment in SLE. The mouse model demonstrates selective effects of DNRAb on hippocampal neurons following blood brain barrier (BBB) breach. ${ }^{1}$ We previously identified abnormal hippocampal glucose hypermetabolism in SLE patients that correlated with serum DNRAb titers and poor performance on neuropsychological (NP) testing. ${ }^{2}$ However, little is known about how antibodies access brain in humans. We evaluated BBB permeability (BBBP) in SLE and healthy control (HC) subjects with DCE-MRI and hypothesized that regions with abnormal hypermetabolism would also demonstrate altered BBBP.

Methods 6 SLE subjects with no history of NP symptoms and 6 age and gender matched HCs underwent NP testing using the Automated Neuropsychological Assessment Metric (ANAM) computerized battery and DCE-MRI on a 3.0 tesla magnet. MRI sequences were acquired according to standard protocols; permeability imaging used DCE technique with axial 3D-SPGR T1-WI sequences and 80 cine phases using $\mathrm{TR}=25 \mathrm{~ms}, \mathrm{TE}=3.8 \mathrm{~ms}, \mathrm{FOV}=24 \mathrm{~mm}$, and matrix size of $128 \times 256$. Magnevist Gadolinium contrast, $0.1 \mathrm{mmol} / \mathrm{kg} \mathrm{IV}$, was dosed at $5 \mathrm{cc} / \mathrm{sec}$ following a $5 \mathrm{sec}$ delay. Post-processing of images into BBBP parameters of K-trans $(\mathrm{mL} / 100 \mathrm{gm} / \mathrm{min}$ ) and VE (mL/100 gm) was performed using Olea Sphere 2.2 and 2.3 with the Tofts extended permeability model. This 